\title{
A molecular systems approach to modelling human skin pigmentation: identifying underlying pathways and critical components
}

\author{
Arathi Raghunath ${ }^{1 \dagger}$, Awanti Sambarey $^{2 \dagger}$, Neha Sharma ${ }^{2}$, Usha Mahadevan ${ }^{1}$ and Nagasuma Chandra ${ }^{2 *}$
}

\begin{abstract}
Background: Ultraviolet radiations (UV) serve as an environmental stress for human skin, and result in melanogenesis, with the pigment melanin having protective effects against UV induced damage. This involves a dynamic and complex regulation of various biological processes that results in the expression of melanin in the outer most layers of the epidermis, where it can exert its protective effect. A comprehensive understanding of the underlying cross talk among different signalling molecules and cell types is only possible through a systems perspective. Increasing incidences of both melanoma and non-melanoma skin cancers necessitate the need to better comprehend UV mediated effects on skin pigmentation at a systems level, so as to ultimately evolve knowledge-based strategies for efficient protection and prevention of skin diseases.

Methods: A network model for UV-mediated skin pigmentation in the epidermis was constructed and subjected to shortest path analysis. Virtual knock-outs were carried out to identify essential signalling components.

Results: We describe a network model for UV-mediated skin pigmentation in the epidermis. The model consists of 265 components (nodes) and 429 directed interactions among them, capturing the manner in which one component influences the other and channels information. Through shortest path analysis, we identify novel signalling pathways relevant to pigmentation. Virtual knock-outs or perturbations of specific nodes in the network have led to the identification of alternate modes of signalling as well as enabled determining essential nodes in the process.

Conclusions: The model presented provides a comprehensive picture of UV mediated signalling manifesting in human skin pigmentation. A systems perspective helps provide a holistic purview of interconnections and complexity in the processes leading to pigmentation. The model described here is extensive yet amenable to expansion as new data is gathered. Through this study, we provide a list of important proteins essential for pigmentation which can be further explored to better understand normal pigmentation as well as its pathologies including vitiligo and melanoma, and enable therapeutic intervention.
\end{abstract}

Keywords: Systems perspective, Skin, UV, Melanin, Networks

\footnotetext{
* Correspondence: nchandra@biochem.iisc.ernet.in

'Equal contributors

${ }^{2}$ Department of Biochemistry, Indian Institute of Science, Bangalore 560012, India

Full list of author information is available at the end of the article
}

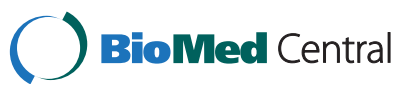

(c) 2015 Raghunath et al.; licensee BioMed Central. This is an Open Access article distributed under the terms of the Creative Commons Attribution License (http://creativecommons.org/licenses/by/4.0), which permits unrestricted use, distribution, and reproduction in any medium, provided the original work is properly credited. The Creative Commons Public Domain Dedication waiver (http://creativecommons.org/publicdomain/zero/1.0/) applies to the data made available in this article, unless otherwise stated. 


\section{Background}

Skin pigmentation is a phenotype evolved and retained over generations, primarily as protection against harmful Ultraviolet (UV) radiations [1]. Melanin, a light-absorbing polymer with photochemical properties imparts colour to human skin and other tissues such as hair and iris, and provides protection against UV induced damage. Variations in skin pigmentation depend on the level and the type of melanin expressed. The two major forms of melanin expressed in humans are eumelanin and pheomelanin, imparting black or brown and red haired or freckled phenotypes respectively. Melanin is synthesized in melanocytes in specialized organelles called melanosomes [2]. Transfer of the pigment to keratinocytes that constitute the upper layers of the epidermis is essential for phenotypic manifestation of skin pigmentation. The dendrites on melanocytes aid in the transfer of melanosomes to keratinocytes where melanosome uptake occurs through phagocytosis [2].

Exposure to UV radiation triggers signalling cascades in keratinocytes, which in turn induce autocrine signalling as well as paracrine signalling with neighbouring melanocytes resulting in melanogenesis, and thereby subsequent processes leading to skin pigmentation. While UV radiations trigger pigmentation, they are also capable of causing skin cancer due to the generation of free radicals and DNA damage, which is actively prevented by the 'epidermal melanin unit' - the association of one melanocyte with several surrounding epidermal keratinocytes [3]. UV exposure can also lead to the depletion of folic acid, though a certain amount of exposure is essential for Vitamin D synthesis [4]. Hence, a balanced exposure to UV radiation is critical, and is maintained by the regulation of melanin synthesis. Constitutive skin pigmentation is determined by the genetic constitution of the individuals while facultative pigmentation is governed by environmental factors [5]. Oxidation and re-distribution of melanin in skin induces some marginal protection but delayed and sustained tanning response to UV includes melanin synthesis and transport to keratinocytes [6].

The process of UV mediated skin pigmentation is complex and dynamic, involving various cell types and extensive cross-talk between them as well as several intracellular signalling cascades. While several studies over the past few decades have provided insights into melanogenesis, melanosome formation and pigmentation [7-9] their focus has been primarily on individual reactions and molecular changes. The World Health Organization reports incidences of between 2 and 3 million non-melanoma skin cancers and more than 100,000 melanoma skin cancers globally each year [10]. These statistics highlight the need for further studies in this area, and a wholistic understanding of pigmentation is thus necessary.

To this end, a network approach is useful as networks have emerged as an important tool to study biological phenomena [11]. The 'omics' era has resulted in the generation of enormous biological data, from individual gene and protein sequences and structures to welldefined physical and functional interactions and elaborate signalling cascades. By integrating this information of communication between various molecular components in the form of an interaction network, biological processes can be represented and analysed at a systems level, wherein the contributions and effects of individual components and interactions on the entire system can be studied to generate insights which cannot be achieved by reductionist approaches alone. Extensive research in the field of skin pigmentation has resulted in vast accumulation of data, but studies that integrate and analyse this data are limited [12-14].

In this study, we curate data available from literature and construct a comprehensive network model of UV mediated pigmentation in both keratinocytes and melanocytes, in an attempt to provide mechanistic insights into signalling cascades underlying skin pigmentation. Further, we study the effects of knock-outs of certain components on these signalling pathways, leading to the identification of essential genes as well as highlight key destinations and possible alternative signalling routes in the process of pigmentation.

\section{Results}

A comprehensive network model of human pigmentation We have constructed a network model of human skin pigmentation based on extensive data available from literature. The model includes various components of (a) melanin biosynthesis, (b) melanosome biogenesis, (c) dendrite formation and (d) uptake of melanosomes by keratinocytes. Such a comprehensive systems-level picture of skin pigmentation has the potential to address several questions, particularly the critical nature of various factors involved in pigmentation and its response to UV radiation.

Several experimental studies have been referred to for network construction as enlisted in Additional file 1. However, for clarity, a brief description of the model and its components is given below.

Melanin, the photosensitive polymer that imparts skin pigmentation is synthesised and stored in melanosomes organelles specific to melanocytes [7]. Melanin is synthesised in the body from the amino acid tyrosine, through a series of biochemical reactions primarily involving the enzymes tyrosinase, DOPAchrome tautomerase and tyrosinase-related protein 1 (TYRP1). Tyrosine is first oxidized to DOPA and subsequently to DOPAquinone by the enzyme tyrosinase. DOPAquinone can be enzymatically converted to either eumelanin or pheomelanin. During eumelanin synthesis, DOPAquinone is converted to leucoDOPA and subsequently to DOPAchrome by autooxidation. DOPAchrome tautomerase, in the presence of 
dihydroxyindole-2-carboxylic acid oxidase, converts DOPAchrome to 5,6-dihydroxy indole. Tyrosinase acts on 5,6-hydroxy indole finally resulting in eumelanin synthesis. An alternate biosynthesis pathway leading to pheomelanin involves conversion of DOPAquinone to cysteinylDOPA in presence of glutathione/cysteine, followed by oxidative polymerization of the benzothiazinylalanine intermediates, resulting in pheomelanin synthesis [15].

Though melanin biosynthesis is a central process in skin pigmentation, multiple associated pathways are necessary for successful manifestation of the phenotype. In response to the necessary UV stimulus, melanocytes form dendrites, and through them transport melanin carrying melanosomes to the peripheral keratinocytes where the melanosomes are subjected to phagocytosis, resulting in the expression of melanin in keratinocytes, ultimately leading to skin pigmentation [7].

Here, we present an extensive systems model of pigmentation that includes interactions and pathway networks involved in all the major biological processes discussed above leading to the pigmentation of skin, beginning with melanosome formation and melanogenesis and culminating in its expression in keratinocytes leading to skin pigmentation. Figure 1 provides a schematic representation of the complex network of the various processes leading to human skin pigmentation. The model is depicted in the form of a static map that is compartmentalised to depict several signalling events and flow of signal information in keratinocytes and melanocytes on exposure to UV. As can be observed, the model captures several signalling cascades triggered in keratinocytes on initial exposure such as lipid peroxidation, MAPK signalling, TNF-receptor mediated skin inflammation and other processes which result in melanogenesis in melanocytes via extensive cross-talk. The model also contains a detailed molecular description of the synthesis of melanin in melanosomes, followed by dendritogenesis which enables melanosome phagocytosis by keratinocytes, where melanin finally manifests itself in the form of the skin pigment. The model also includes additional pathways stimulated by UV radiation like skin ageing, cell proliferation and differentiation and DNA damagedminimised by the process of DNA repair, mediated by melanin. While the model provides a comprehensive description of skin pigmentation, given the vastness of research in this field and increasing data availability, it lends itself to incorporation of additional data as and when it becomes available.

Based on the signalling events described in the model, 265 participating molecules were identified, including 189 proteins, 33 small molecules/compounds, 23 complexes, 18 biological processes and 2 environmental factors. The model thus incorporates multiple levels of detail. For these 265 molecules or nodes, interactions were extracted as described in Methods, and a network was constructed comprising 265 nodes and 429 edges. The edges were assigned direction by extensive literature curation, highlighting the functional nature of each interaction. 142 nodes were involved in keratinocyte signalling, while 113 nodes contributed to signalling within melanocytes, and these were ascribed the suffix 'kerat' and 'melan' respectively. The complete list of all nodes and edges in the network along with the nature and direction of their interactions are provided in Additional file 1. A sample network description has been provided in Table 1. This network was visualized in Cytoscape v3.1.0 and several network properties were computed based solely on network topology. As the network was directed, both in-degree and out-degree distributions were computed describing their connectivity and certain 'hub' nodes which are central to the network were thus identified. All network properties of degree distributions, identified hubs, node centrality, edge centrality and radiality computed are described in Additional file 2.

\section{Identifying biochemical routes in the network among defined sources and targets}

The constructed network provides a basis for understanding communication and signalling within the system. A systems level view of pigmentation provides insights into how information flows via signalling cascades both at inter-and intra cellular levels, and encompasses cross talk among different molecules and cell types. One approach towards understanding network dynamics and communication is through the analysis of shortest paths between pairs of nodes, as demonstrated in several studies in different biological conditions [16,17]. A total of 33,779 all-vsall paths for the 265 nodes were computed. For a more definitive analysis of pathways of interest in the process of pigmentation, specific source and destination nodes were chosen and paths from these sources to destinations were inspected in detail. The initiating nodes or sources included key receptors and triggers such as UV radiation. 20 source nodes were identified, based on their likelihood of initiating signalling events. All sources culminated in biological end processes such as melanosome formation, melanogenesis, dendrite formation and melanosome phagocytosis, among others. A total of 9 destinations were thus shortlisted. Table 2 enlists the selected source and target nodes in the network. Between these 20 sources and 9 destination nodes, 157 paths were computed which were further analyzed. The source to target (S2T) sub-network had 127 nodes and 150 edges, and the resulting network was completely interconnected, with no independent interactions. This indicates a well-knit network with extensive signalling crosstalk. The 127 nodes were seen to participate in various biological processes in both keratinocytes and melanocytes including cell differentiation, proliferation, survival, cell cycle arrest, apoptosis, melanosome 


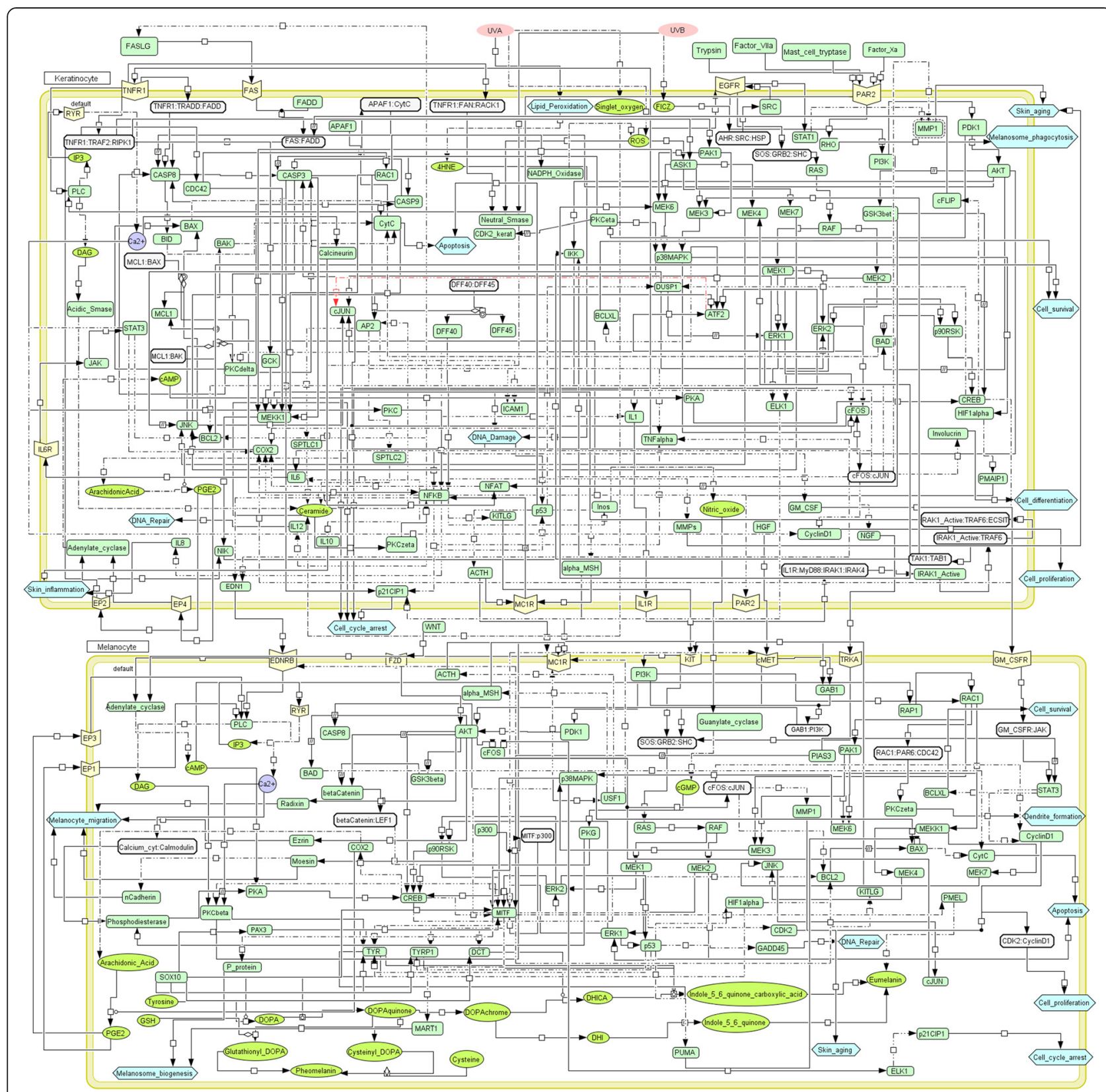

Figure 1 Schematic representation of signalling events triggered in skin cells upon exposure to UV radiation. The two compartments in the image represent the two cell types- keratinocyte and melanocyte. Generic proteins are represented in rectangular boxes while receptors are specifically shown on the membrane; biological processes are represented as hexagons, and secondary messengers and other small molecules involved in the signaling network as ellipses and circles. The environmental triggers UVA and UVB are represented as pink ellipses. Complexes of two or more proteins are also depicted in rectangles, with the proteins involved in the complex separated by a colon (:). The arrows describe the nature of interaction: activation is depicted as $\rightarrow \longrightarrow$, inhibition as $\rightarrow-$, expression as $\cdots \square-$ and third molecule regulation as $\downarrow$. The schema also describes paracrine (reactions occurring within a compartment) and autocrine (reactions occurring cross-compartmentally) signalling.

biogenesis, eumelanin and pheomelanin synthesis, melanocyte migration, dendrite formation, melanosome phagocytosis, skin inflammation and skin ageing, thus covering and connecting several signalling cascades, as enlisted in Additional file 3. This S2T sub network was visualized in Cytoscape and is depicted in Figure 2. All computed paths from the selected source to target nodes are listed in Additional file 4.

\section{Identification of novel signalling pathways}

Of the 157 computed paths between the desired source and target nodes, a few of them were shown to be well 
Table 1 A sample Protein-Protein interaction network constructed in this study

\begin{tabular}{|c|c|c|}
\hline Node A & Node B & $\begin{array}{l}\text { Interaction type } \\
\text { A-> B }\end{array}$ \\
\hline$\overline{U V A}$ & Lipid_Peroxidation_kerat & induces \\
\hline Lipid_Peroxidation_kerat & 4HNE_kerat & increases level \\
\hline 4HNE_kerat & DNA_Damage_kerat & induces \\
\hline DNA_Damage_kerat & TP53_kerat & activates \\
\hline TP53_kerat & ACTH_kerat & $\begin{array}{l}\text { increases } \\
\text { expression }\end{array}$ \\
\hline TP53_kerat & alpha_MSH_kerat & $\begin{array}{l}\text { increases } \\
\text { expression }\end{array}$ \\
\hline ACTH_kerat & MC1R_melan & activates \\
\hline alpha_MSH_kerat & MC1R_melan & activates \\
\hline MC1R_melan & ADCY4_melan & activates \\
\hline ADCY4_melan & CAMP_melan & increases level \\
\hline CREB1_melan & PTGS2_melan & $\begin{array}{l}\text { increases } \\
\text { expression }\end{array}$ \\
\hline PGE2_kerat & PTGER3_melan & activates \\
\hline PGE2_kerat & PTGER1_melan & activates \\
\hline PTGER3_melan & PLC_melan & activates \\
\hline PTGER1_melan & PLC_melan & activates \\
\hline PLC_melan & DAG_melan & increases level \\
\hline DAG_melan & PRKCB_melan & activates \\
\hline PTGS2_melan & PGE2_melan & Increases level \\
\hline PRKCB_melan & TYR_melan & activates \\
\hline TYR_melan & Eumelanin_melan & Increases level \\
\hline
\end{tabular}

The complete network can be found in Additional file 1.

characterised in literature, thus serving to validate our analysis. Signalling through Protein Kinase A (PKA) culminating in melanogenesis has been well established. Also, reports of melanocyte stimulating hormone (alphaMSH/MC1R) mediated melanogenesis involving PKA/CREB signalling have been elucidated in a few individual studies $[18,19]$. Through our study, we identified the same pathway and traced it to MSH as the source. Such a study helps us to systematically relate individual genes to particular processes.

Another example pathway identified in our network describes the stem cell factor (KITLG) mediated activation of MITF, a primary transcription factor that regulates expression of melanogenic proteins.

Several novel signalling cascades showing possible paracrine signalling between keratinocytes and melanocytes were predicted by the network. As an example, some of the predicted UV mediated paths that lead to melanin synthesis, dendrite formation, phagocytosis and melanocyte proliferation are described in Table 3. Certain observations from literature are also provided which
Table 2 Nodes selected as source and target for shortest path analysis

\begin{tabular}{ll}
\hline Source nodes & Target nodes \\
\hline MET_melan & Eumelanin_melan \\
EDNRB_melan & Pheomelanin_melan \\
EGFR_kerat & Dendrite_formation_melan \\
PTGER1_melan & Melanosome_phagocytosis_kerat \\
PTGER2_kerat & Apoptosis_melan \\
PTGER3_melan & Cell_proliferation_melan \\
PTGER4_kerat & Melanosome_biogenesis \\
FAS_kerat & Cell_survival_melan \\
FZD3_melan & Cell_cycle_arrest_melan \\
CSF2RA_melan & \\
IL1R1_kerat & \\
IL6R_kerat & \\
KIT_melan & \\
MC1R_kerat & \\
MC1R_melan & \\
F2RL1_kerat & \\
TNFRSF1A_kerat & \\
NTRK1_melan & \\
UVA & \\
UVB & \\
\hline
\end{tabular}

could possibly validate the existence of such biological routes.

Perturbation networks: identifying 'essential nodes'

Interaction networks help identify important nodes based on their connections and describe the topological architecture of a system. In order to assess the relative importance of a node, the following criteria was used: a) node degree, leading to the identification of hubs in the network (b) frequency of occurrence of a given node in all shortest paths - the more frequently a node occurs, the greater is its importance in the system as it is an important mediator in signalling from several sources to their respective destinations.

Based on these criteria, 33 nodes were shortlisted as most important or significant in the network. While this result is significant in itself, our objective was to identify the functional effects of these molecules in the process of pigmentation. These nodes were then knocked-out and 33 different perturbation networks were constructed, with each network specific to a perturbation wherein a selected node and its connecting edges were removed. Shortest paths from the same 20 sources to the 9 destinations were computed for each perturbed network and analyzed to identify all plausible paths leading to a similar end process. 




Perturbation studies served to address the following questions: (a) does a signal from a given source reach its destination if the node mediating its pathway is perturbed? and (b) do alternate paths exist from a particular source to a destination that can be utilised upon perturbation? While some perturbations resulted in the complete lack of signalling from a given source to a target, several others resulted in signalling via alternate routes. Nodes whose absence resulted in abrogation of pathways were considered as 'essential nodes'. In certain cases, two or more nodes are seen to be essential for a given pathway. Table 4 shortlists these essential nodes, rank listed in order of the number of pathways they disrupt, and these nodes are also highlighted in Figure 2. A few examples of alternate routes upon perturbation are provided in Table 5, and a few others illustrated in Figure 3. Studies from literature supporting the possibility of such alternate paths have also been cited, and none of these studies overlap with the literature referred to while building the model, thereby providing greater credibility to our findings. All perturbation results describing essential nodes as well as alternate paths have been provided in Additional file 5.

\section{New insights from the model}

Data from the network shows the influence of signalling from multiple receptors in processes leading to pigmentation. Significant contributors to skin pigmentation have been identified by the model, with several receptors seen to be playing a key role. Receptors EGFR, F2RL1, FAS, IL1R1, IL6R, PTGER2, PTGER4 and TNFRSF1A in keratinocytes, and CSF2RA, EDNRB, FZD3, KIT, MET,
NTRK1, PTGER1 and PTGER3 in melanocytes are observed to be important for pigmentation. MC1R signalling is seen to be important in both keratinocytes and melanocytes. Some of the paths in the model corroborate with knockout and siRNA data that emphasize the need for certain proteins for the manifestation of the skin pigmentation phenotype and their role in various skin pathologies. The model also highlights paracrine signalling in cells of the epidermis wherein factors secreted from keratinocytes in response to UV have a key role to play in adult skin pigmentation by regulating signalling in melanocytes. The model to a large extent encompasses signalling involving various physiological factors that are known to play a role in adult skin pigmentation such as MC1R, CREB, MITF, PAX3, SOX10, LEF1/TCF, F2RL1, SCF, HGF and GM-CSF [15].

\section{Discussion}

Human skin responds to UV radiation exposure by increasing the expression of melanin in skin, a pigment that protects it from UV induced damage [6]. Melanin has a role to play in various pigmentation disorders such as albinism [20], vitiligo [21] as well as in melanoma [22] and nonmelanoma skin cancers [23]. A detailed understanding of the process of skin pigmentation is thus vital for understanding the molecular pathology of these disease conditions. Melanin synthesis takes place in response to UV induced cues from neighbouring cells such keratinocytes and dermal fibroblasts [24], and exerts its protective effects when transported and expressed in keratinocytes [25]. Multiple roles are suggested for the protective effect of melanin 
Table 3 Example predicted pathways from source to targets

\begin{tabular}{|c|c|}
\hline $\begin{array}{l}\text { Biological } \\
\text { process }\end{array}$ & UV mediated melanogenesis [Tyrosinase activation]-paracrine effect \\
\hline $\begin{array}{l}\text { Predicted } \\
\text { pathway }\end{array}$ & $\begin{array}{l}\text { UV- > Singlet_oxygen_kerat- > Ceramide_kerat- > PLA2_kerat- > Arachidonic_Acid_kerat- > PGE2_kerat- > PTGER3_melan- > PLC_melan- > } \\
\text { DAG_melan- > PRKCB_melan- > TYR_melan- > Eumelanin_melan/ Pheomelanin_melan }\end{array}$ \\
\hline Validation & $\begin{array}{l}\text { PGE2 acts as a ligand for PTGER3 receptor. COX2 is the enzyme involved in the conversion of arachidonic acid to PGE2. UV induced COX2 } \\
\text { and increased PGE2 production in keratinocytes [42-45]. }\end{array}$ \\
\hline $\begin{array}{l}\text { Predicted } \\
\text { pathway }\end{array}$ & UV- > Singlet_oxygen_kerat- > Ceramide_kerat- > PRKCZ_kerat- > NFKB1_kerat- > PTGS2_kerat \\
\hline Validation & Antioxidants like Astaxanthin are shown to inhibit UV induced PGE2 production possibly by down regulating COX2 expression [46]. \\
\hline $\begin{array}{l}\text { Biological } \\
\text { process }\end{array}$ & UV mediated melanogenesis [Tyrosinase expression]-paracrine effect \\
\hline $\begin{array}{l}\text { Predicted } \\
\text { pathway }\end{array}$ & $\begin{array}{l}\text { UV- > Singlet_oxygen_kerat- > Ceramide_kerat- }>\text { PRKCZ_kerat- }>\text { NFKB1_kerat- }>\text { KITLG_kerat }->\text { KIT_melan- }>\text { PIK3CA_melan- }> \\
\text { PDPK1_melan- }>\text { AKT1_melan- }>\text { CREB1_melan- }>\text { MITF_melan- > TYR_melan- }>\text { Eumelanin_melan/ Pheomelanin_melan }\end{array}$ \\
\hline Validation & KIT mediated regulation of MITF activity is shown to involve PI3KAKT signaling [47]. \\
\hline $\begin{array}{l}\text { Predicted } \\
\text { pathway }\end{array}$ & $\begin{array}{l}\text { UVA- > Singlet_oxygen_kerat- }>\text { Ceramide_kerat- }>\text { PRKCZ_kerat- }>\text { NFKB1_kerat- }>\text { NOS2_kerat- }>\text { Nitric_oxide_kerat- }> \\
\text { GUCY1A2_melan- > CGMP_melan- > PRKG1_melan- > CREB1_melan- > MITF_melan- > TYR_melan- }>\text { melanogenesis }\end{array}$ \\
\hline Validation & $\begin{array}{l}\text { C-phycocyanin, a phycobiliprotein from spirulina that has antioxidant function inhibits melanogenesis by decreasing activity of } \\
\text { CREB and suppressing tyrosinase expression [48]. Compound, MHY498 inhibited sodium nitroprusside (SNP, a NO donor)-induced } \\
\text { NO generation, and suppressed tyrosinase expression, MITF stimulation and melanin synthesis through CGMP-mediated signaling } \\
\text { pathway in B16F10 melanoma cells [49]. }\end{array}$ \\
\hline $\begin{array}{l}\text { Biological } \\
\text { process }\end{array}$ & UV mediated alpha MSH production \\
\hline $\begin{array}{l}\text { Predicted } \\
\text { pathway }\end{array}$ & UVA- > Lipid_Peroxidation_kerat- > 4HNE_kerat- > DNA_Damage_kerat- > TP53_kerat- > alpha_MSH_kerat \\
\hline Validation & $\begin{array}{l}\text { UV releases singlet oxygen upon lipid peroxidation [50]. N-acetylcysteine with antioxidant properties can suppress alphaMSH and } \\
\text { ACTH production in response to UV [51]. AlphaMSH is synthesised in keratinocytes in a p53 dependent manner [52]. }\end{array}$ \\
\hline $\begin{array}{l}\text { Biological } \\
\text { process }\end{array}$ & UV mediated melanocyte dendrite formation-paracrine effect \\
\hline $\begin{array}{l}\text { Predicted } \\
\text { pathway }\end{array}$ & $\begin{array}{l}\text { UV- > Singlet_oxygen_kerat- > Ceramide_kerat- }>\text { PRKCZ_kerat- }>\text { NFKB1_kerat- }>\text { KITLG_kerat- }>\text { KIT_melan- }>\text { PIK3CA_melan- > } \\
\text { RAC1_melan- > RAC1:PARD6A:CDC42_melan- }>\text { PRKCZ_melan- > Dendrite_formation_melan }\end{array}$ \\
\hline Validation & $\begin{array}{l}\text { UVB exposure increased tree branch-like dendrites and activated Rac1 in a time-dependent manner in B16 melanoma cells [53]. Transiently } \\
\text { expressed Rac1 activated mutants induces the formation of dendrite-like structures in human melanoma cell lines [34]. }\end{array}$ \\
\hline $\begin{array}{l}\text { Biological } \\
\text { process }\end{array}$ & UV mediated NFKB1 activation and secretion of paracrine factors \\
\hline $\begin{array}{l}\text { Predicted } \\
\text { pathway }\end{array}$ & UV-> Singlet_oxygen_kerat- > Ceramide_kerat- > PRKCZ_kerat- > NFKB1_kerat- > EDN1_kerat \\
\hline $\begin{array}{l}\text { Predicted } \\
\text { pathway }\end{array}$ & UV- > Singlet_oxygen_kerat- > Ceramide_kerat- > PRKCZ_kerat- > NFKB1_kerat- > CSF2_kerat \\
\hline $\begin{array}{l}\text { Predicted } \\
\text { pathway }\end{array}$ & UV- > Singlet_oxygen_kerat- > Ceramide_kerat- > PRKCZ_kerat- > NFKB1_kerat- > KITLG_kerat \\
\hline Validation & UV is known activate NFkappaB in keratinocytes $[54,55]$. \\
\hline
\end{tabular}

Biological process

UV mediated melanocyte proliferation-paracrine effect

Predicted pathway

UVA-> Singlet_oxygen_kerat- > Ceramide_kerat- > PRKCZ_kerat- > NFKB1_kerat- > CSF2_kerat- > CSF2RA_melan- > CSF2RA:JAK1_melan- > STAT3_melan- > CCND1_melan- > CDK4:CCND1_melan- > Cell_proliferation_melan

Validation Korean Red Ginseng extract or its saponin (KRGE or SKRG) decreased the expression of CSF2 in keratinocytes induced by UVB irradiation and also decreased proliferation of melanocytes [56].

Biological process

UV induced melanosome phagocytosis

Predicted pathway 
Table 3 Example predicted pathways from source to targets (Continued)

\begin{tabular}{ll}
\hline Validation & In a co-culture system model constructed using the primary human melanocytes and keratinocytes, increased melanosome transfer and \\
& also upregulation of F2RL1 protein in the keratinocytes is observed when treated with low concentrations of H(2)O(2) [57]. Madecassoside \\
& (MA), a pentacyclic triterpene significantly inhibited UVR-induced melanin synthesis and melanosome transfer in a co culture system of \\
keratinocytes and melanocytes and also inhibited F2RL1 expression in keratinocytes [58].
\end{tabular}

Validation refers to observations from literature that could possibly support the existence of such pathways.

in UV induced damage [26]. Melanin can filter UV radiation and scavenge reactive oxygen species produced as a result of UV exposure. The supra-nuclear cap of melanin protects cells from DNA damage. It is reported to inhibit production of cyclobutane pyrimidine dimers and 6,4-photoproducts, both of which are mutagenic [27]. Patients with albinism have been shown to be more susceptible to various skin cancers [28].

Given the fact that manifestation of the phenotype involves a complex cross-talk across different cell types that are present in different strata of the skin through a well regulated sequence of events, it becomes imperative to study the process from a systems perspective. The availability of data through several reductionist experimental studies over the years has greatly accelerated advances in systems' analyses of pigmentation [29]. A recent study integrated functional genomics and protein-protein interaction networks to identify novel components that impact melanogenesis, focusing largely on the endothelin receptor (EDNRB) mediated signalling pathway [30]. Our study also supports the importance of ENDRB mediated signalling in melanocytes in the process of melanogenesis.

Our model describes a well annotated, curated and directed interaction network among 265 components that could have a direct or indirect role in influencing skin

\begin{tabular}{ll}
$\begin{array}{l}\text { Table } 4 \text { Essential nodes - Nodes are ranked according to } \\
\text { the number of source- }>\text { target pathways they are } \\
\text { essential in }\end{array}$ & $\begin{array}{l}\text { No: of source- }>\text { target pathways } \\
\text { it is essential in }\end{array}$ \\
\hline Node & 34 \\
\hline RAC1_melan & 29 \\
MITF_melan & 25 \\
NFKB1_kerat & 18 \\
TYR_melan & 14 \\
PLC_melan & 9 \\
PTGS2_kerat & 9 \\
CASP8_kerat & 8 \\
F2RL1_kerat & 7 \\
AKT1_melan & 7 \\
ADCY4_melan & 4 \\
CREB1_melan & 2 \\
PRKCD_kerat & 1 \\
PTGER1_melan & 1 \\
MC1R_melan &
\end{tabular}

pigmentation. This is the first study reporting a directed interaction network of human skin pigmentation, highlighting the functional nature of interactions among different components, as well as the direction in which a signal has to flow to be biologically meaningful. Of the 265 nodes, 183 have a role to play in regulating more than one biological process influencing skin pigmentation. This reflects the complexity as well as the connectivity within the system where a single node can influence multiple biological processes. The model suggests that multiple cues from neighbouring cells can trigger similar processes in melanocytes, highlighting the various possibilities of multiple alternate paths a system could take leading to a common end process. The importance of certain nodes has been illuminated, some of which are well known, such as the indispensability of tyrosinase in melanogenesis [31] and the role of MITF in melanosome formation [32]. We also report a list of nodes that are essential for certain processes, the absence of which leads to abrogation of signalling through the required pathway. The model helps in broadening the perspective of known data by suggesting an extended signalling pathway or potential alternate paths in the absence of a node. F2RL1 is known to play an important role in melanosome transfer [33]. The model suggests that NFKB could be the transcription factor regulating the expression of F2RL1 in keratinocytes and hence NFKB could also have a key role to play in melanosome transfer. RAC1 is involved in MSH mediated dendrite formation on melanocytes [34]. The current model predicts the possibility of an alternate path involving paracrine signalling of KITLG from keratinocytes acting on the c-kit receptor on melanocytes leading to RAC1 activation and subsequent dendrite formation. The model reiterates the communication between keratinocytes and melanocytes.

These observations highlight the need to study biological processes in the context of the system as a whole instead of individual entities, as varied factors both external and internal have a role to play in their regulation. Perturbation studies with individual source-destination pairs enable the identification of components that are essential for a biological process or its phenotypic manifestation. It also hints at the possible dispensable nature of certain proteins and redundancy in the network by suggesting alternate signalling routes.

The vastness and constant addition of data in this field impose a practical limitation on model completeness. Though the network is extensive, it can be further 
Table 5 Alternate paths taken up post perturbation

\begin{tabular}{|c|c|}
\hline $\begin{array}{l}\text { Biological process } \\
\text { perturbed gene }\end{array}$ & UV induced melanin synthesis PTGER3_melan \\
\hline Control Path & $\begin{array}{l}\text { UV- > Singlet_oxygen_kerat- > Ceramide_kerat- > PLA2_kerat- > Arachidonic_Acid_kerat- > PGE2_kerat- > } \\
\text { PTGER3_melan- > PLC_melan- > DAG_melan- > PRKCB_melan- > TYR_melan- > Melanogensis }\end{array}$ \\
\hline Alternate Path & $\begin{array}{l}\text { UV- > Singlet_oxygen_kerat- > Ceramide_kerat- > PRKCZ_kerat- > NFKB1_kerat- > EDN1_kerat- > EDNRB_melan- > } \\
\text { PLC_melan- > DAG_melan- > PRKCB_melan- > TYR_melan- > Eumelanin_melan }\end{array}$ \\
\hline Validation & Addition of EDN1 induced increase in tyrosinase activity in cultured human melanocytes [59]. \\
\hline \multirow{2}{*}{$\begin{array}{l}\text { Biological Process } \\
\text { Perturbed gene }\end{array}$} & UV induced melanin synthesis \\
\hline & Tyr_melan \\
\hline Control Path & $\begin{array}{l}\text { UV- > Singlet_oxygen_kerat- > Ceramide_kerat- > PLA2_kerat- > Arachidonic_Acid_kerat- > PGE2_kerat- > } \\
\text { PTGER3_melan- > PLC_melan- > DAG_melan- > PRKCB_melan- > TYR_melan- }>\text { Melanogensis }\end{array}$ \\
\hline Alternate Path & None \\
\hline Validation & Tyrosinase mutations lead to albinism or hypopigmentation (MGI) \\
\hline \multirow{2}{*}{$\begin{array}{l}\text { Biological Process } \\
\text { Perturbed gene }\end{array}$} & UV induced dendrite formation \\
\hline & KIT_melan \\
\hline Control Path & $\begin{array}{l}\text { UV- }>\text { Singlet_oxygen_kerat- }>\text { Ceramide_kerat- }>\text { PRKCZ_kerat- }>\text { NFKB1_kerat- }>\text { KITLG_kerat- }>\text { KIT_melan- }> \\
\text { PIK3CA_melan- }>\text { RAC1_melan- }>\text { RAC1:PARD6A:CDC42_melan- }>\text { PRKCZ_melan- }>\text { Dendrite_formation_melan }\end{array}$ \\
\hline Alternate Path & $\begin{array}{l}\text { UVB- > Lipid_Peroxidation_kerat- }>\text { 4HNE_kerat- }>\text { DNA_Damage_kerat- }>\text { TP53_kerat- }>\text { ACTH_kerat- }>\text { MC1R_melan- }> \\
\text { ADCY4_melan- > cAMP_melan- > RAP1A_melan- > RAC1_melan- }>\text { RAC1:PARD6A:CDC42_melan- }>\text { PRKCZ_melan- }> \\
\text { Dendrite_formation_melan }\end{array}$ \\
\hline Validation & $\begin{array}{l}\text { Highly dendritic melanocytes are stimulated by injection of alpha-MSH in newborn mice. Formation and translocation } \\
\text { of melanosomes to dendrites is triggered by alpha-MSH [60]. }\end{array}$ \\
\hline
\end{tabular}

Biological Process Perturbed gene

Control Path

Alternate Path

Validation

Biological Process Perturbed gene

Control Path

Alternate Path

Validation

\section{UV induced melanosme phagocytosis}

\section{F2RL1_kerat}

UV- > Singlet_oxygen_kerat- > Ceramide_kerat- > PRKCZ_kerat- > NFKB1_kerat- > F2RL1_kerat- > RHOA_kerat- > Melanosome_phagocytosis_kerat

None

RWJ-50353, a serine protease inhibitor, led to reduced pigment deposition in melanocytes and de-pigmentation. Immature melanosomes accumulate inside melanocytes and there is abnormal dendrite dynamics in RWJ-50353treated epidermal equivalents [61].

\section{UV induced melanocyte proliferation}

\section{NFKB1_kerat}

UV-> Singlet_oxygen_kerat - > Ceramide_kerat- > PRKCZ_kerat- > NFKB1_kerat- > CSF2_kerat- > CSF2RA_melan- > CSF2RA:JAK1_melan->STAT3_melan- >CCND1_melan- >CDK4:CCND1_melan- >Cell_proliferation_melan

UVB- > Lipid_Peroxidation_kerat- > 4HNE_kerat- > DNA_Damage_kerat- $>$ TP53_kerat- $>$ ACTH_kerat- > MC1R_melan- > ADCY4_melan- $>$ CAMP_melan- $>$ PRKACA_melan- $>$ CREB1_melan- $>$ MITF_melan- $>$ CDK4_melan- $>$

CDK4:CCND1_melan->Cell_proliferation_melan

In T-oligos pre-treated and UV light-irradiated keratinocytes, NFKB binding to the transcriptional co-activator p300 decreased relative to control whereas the amount of p53 binding to p300 was strikingly increased demonstrating activation of p53 and repression of NFkB upon DNA damage in keratinocytes which could serve as an alternate path when NFkB is perturbed [62].

Validation refers to observations from literature that could possibly support the existence of such pathways.

extended with the availability of more information. The model can also be expanded to include factors from dermal fibroblasts, which are now being reported as increasingly important in the manifestation of pigmentation [35].

Aberrant melanin expression can lead to multiple pathological conditions like albinism - which results in a complete loss of pigmentation, vitiligo - wherein there occur hypo-pigmented patches of skin, sunburn and skin damage due to insufficient protection of the skin from $\mathrm{UV}$ radiation, or melanoma, one of the most aggressive forms of human skin cancer. Skin pigmentation has also been a significant field of interest for the cosmetic industry which attempts to address issues of lentigines/age spots and improving the skin tone. Use of anti-oxidants for correcting pigmentation disorders [36,37] can be readily elucidated from the model as UV induced oxidative stress is one of the primary steps triggering signalling leading to regulation of skin pigmentation. Thus, a foundation of cellular signalling has been provided which can be used to study conditions of disease and provide a basis for identifying therapeutic targets. 


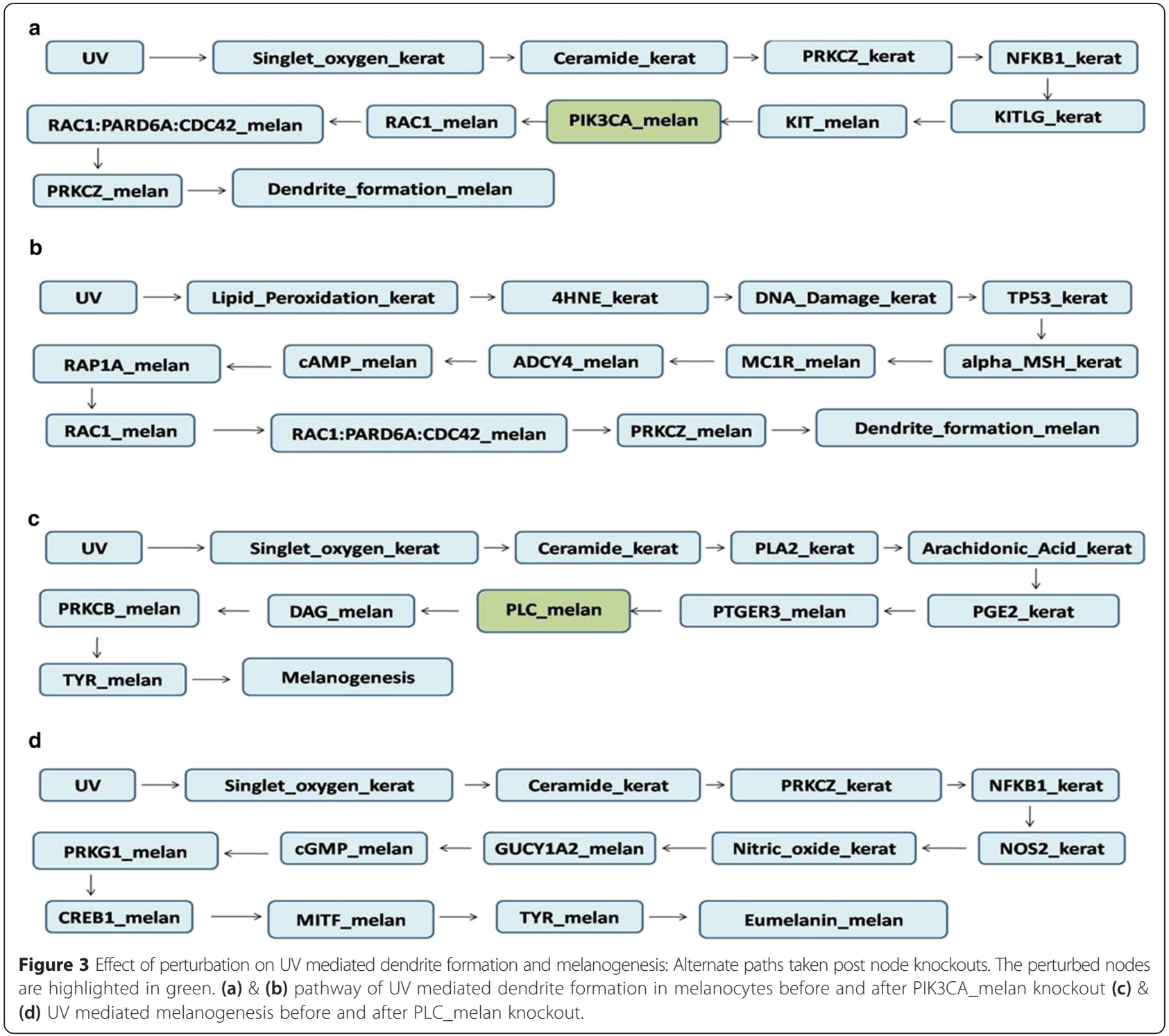

\section{Conclusions}

Through this study we have presented a systems perspective to the process of UV mediated human skin pigmentation, identifying underlying pathways in the process. A comprehensive, well annotated directed interaction network of various molecules participating in the manifestation of skin pigmentation in the epidermis has been constructed. The model has provided a detailed description of signalling pathways triggered in epidermal cells, and sequential signalling in keratinocytes and melanocytes. Several plausible routes of biological signalling in the network have been identified, and the importance of certain components and their effects on signalling pathways has been ascertained. A comprehensive understanding of the molecular events leading to skin pigmentation can thus provide valuable inputs for identifying potential targets for therapeutic consideration as well as a rational basis for design/improvement of commercial products. The approach adopted can be applied to various biological systems and serves as a powerful tool to analyse cross-talk among various biological components.

\section{Methods}

\section{Building a network model}

Through an exhaustive literature survey, different processes in skin pigmentation including melanin biosynthesis, melanosome formation in melanocytes followed by transfer of melanin carrying melanosomes to keratinocytes via dendrites and phagocytosis in keratinocytes were identified. For each of these processes, important participating molecules including proteins, secondary messengers and small molecules were identified. A model describing these processes was built as a static map using the software Cell Designer (version 4.4) [38]. Based on this data, an interaction 
network was constructed for these molecules that participate in different stages leading to pigmentation. In the network, individual proteins and in some cases metabolites form the nodes and interactions among the nodes form the edges. The network included protein-protein, protein-small molecule and protein-biological process interactions. All protein-protein interactions were high confidence interactions extracted manually, or from the STRING database and $\mathrm{NetPro}^{\text {тx }}$ [39]. The protein-small molecule interactions and protein-biological process interactions were curated from literature [approximately 100 articles from PubMed]. The interactions included in the network pertained primarily to human data but data from mouse/rat species was also used to fill in certain missing links. The network included molecular interactions influencing pigmentation in response to UV radiation in two primary epidermal cell types- keratinocytes and melanocytes. Interactions in the network were assigned direction, based on information available in literature and KEGG pathways. Directions were dependent on the functional nature of the interaction, for e.g. 'phosphorylation' or 'activation'. All nodes were named according to their compartment, with the suffix kerat or melan ascribed to them (for e.g. NFKB_kerat), depending on their presence in keratinocytes or melanocytes. This compartment-specific interaction information was manually curated through literature.

\section{Network analysis}

The constructed network was visualized in Cytoscape v.3.1.0 [40]. Network properties of node and edge centrality, degree distributions, radiality, and shortest path lengths were computed using the plugin NetworkAnalyzer. For computing shortest paths, Dijkstra's algorithm implemented in Matlab using the Matlab Boost Graph Library [41] was used. Perturbation analysis for selected nodes was carried out by eliminating the chosen nodes and their corresponding edges specific to each perturbation.

\section{Additional files}

Additional file 1: Directed interaction network of 265 nodes and 429 edges. The functional 563 nature of interaction is also described. Sources from literature describing each interaction have been provided.

Additional file 2: Network properties of the interaction network. Computed network properties of degree, centrality and betweenness have been provided.

Additional file 3: 127 nodes in the S2T sub-network annotated according to the biological processes they participate in or influence.

Additional file 4: Paths from source to target nodes in the S2T sub-network. 157 computed shortest paths from 20 source nodes to 9 destination nodes have been enlisted.

Additional file 5: Paths computed post perturbation analysis and identification of essential nodes. Effects of perturbation of 33 nodes on source to target paths with possible alternate routes are provided. Essential nodes for each path have been enlisted.

Competing interests

The authors declare that they have no competing interests.

\section{Authors' contributions}

Both AR and AS contributed equally to this work. AR and NS curated relevant data from literature and compiled the model. AR and AS constructed the interaction network and performed network analysis including shortest path analysis and perturbation studies. AR and AS wrote the manuscript. UM and NSC conceptualised and supervised the project. All authors read and approved of the manuscript.

\section{Acknowledgements}

We thank the Department of Biotechnology (DBT), Government of India for financial support. Facilities used at the Department of Biochemistry, Indian Institute of Science are duly acknowledged. We thank Deepesh Nagarajan for his help with Figure 1.

\section{Author details}

${ }^{1}$ Molecular Connections Private Limited, Bangalore 560004, India. ${ }^{2}$ Department of Biochemistry, Indian Institute of Science, Bangalore 560012 India.

Received: 8 December 2014 Accepted: 17 April 2015

Published online: 29 April 2015

\section{References}

1. Rees JL, Harding RM. Understanding the evolution of human pigmentation: recent contributions from population genetics. J Invest Dermatol. 2011;132(3 Pt 2):846-53.

2. Seiberg M. Keratinocyte-melanocyte interactions during melanosome transfer. Pigment Cell Res. 2001;14(4):236-42.

3. Gilchrest BA, Park HY, Eller MS, Yaar M. Mechanisms of ultraviolet light-induced pigmentation. Photochem Photobiol. 1996;63(1):1-10.

4. Jablonski NG, Chaplin G. The evolution of human skin coloration. J Hum Evol. 2000;39(1):57-106

5. Yamaguchi $Y$, Brenner $M$, Hearing VJ. The regulation of skin pigmentation. J Biol Chem. 2007;282(38):27557-61

6. Brenner $M$, Hearing VJ. The protective role of melanin against UV damage in human skin. Photochem Photobiol. 2008;84(3):539-49.

7. Costin GE, Hearing VJ. Human skin pigmentation: melanocytes modulate skin color in response to stress. FASEB J. 2007;21(4):976-94.

8. Miyamura Y, Coelho SG, Wolber R, Miller SA, Wakamatsu K, Zmudzka BZ, et al. Regulation of human skin pigmentation and responses to ultraviolet radiation. Pigment Cell Res. 2007;20(1):2-13.

9. Videira IF, Moura DF, Magina S. Mechanisms regulating melanogenesis. An Bras Dermatol. 2013;88(1):76-83.

10. WHO [http://www.who.int/uv/fag/skincancer/en/index1.html].

11. Barabasi A-L, Oltvai ZN. Network biology: understanding the cell's functional organization. Nat Rev Genet. 2004:5(2):101-13.

12. Pawelek JM, Chakraborty AK, Osber MP, Orlow SJ, Min KK, Rosenzweig KE, et al. Molecular cascades in UV-induced melanogenesis: a central role for melanotropins? Pigment Cell Res. 1992;5(5 Pt 2):348-56.

13. D'Orazio J, Jarrett $\mathrm{S}$, Amaro-Ortiz A, Scott T. UV radiation and the skin. Int J Mol Sci. 2013;14(6):12222-48.

14. Maddodi N, Jayanthy A, Setaluri V. Shining light on skin pigmentation: the darker and the brighter side of effects of UV radiation. Photochem Photobiol. 2012;88(5):1075-82.

15. Yamaguchi $Y$, Hearing VJ. Physiological factors that regulate skin pigmentation. Biofactors. 2009;35(2):193-9

16. Ghosh S, Baloni P, Mukherjee S, Anand P, Chandra N. A multi-level multi-scale approach to study essential genes in Mycobacterium tuberculosis. BMC Syst Biol. 2013;7(1):132.

17. Verkhedkar KD, Raman K, Chandra NR, Vishveshwara S. Metabolome based reaction graphs of $M$. tuberculosis and $M$. Leprae: a comparative network analysis. PLoS One. 2007;2(9):e881. 
18. Abdel-Malek Z, Scott MC, Suzuki I, Tada A, Im S, Lamoreux L, et al. The melanocortin-1 receptor is a key regulator of human cutaneous pigmentation. Pigment Cell Res. 2000;13 Suppl 8:156-62.

19. Busca R, Ballotti R. Cyclic AMP a key messenger in the regulation of skin pigmentation. Pigment Cell Res. 2000;13(2):60-9.

20. Spritz RA. Molecular genetics of oculocutaneous albinism. Hum Mol Genet. 1994;3:1469-75.

21. Roy S. Melanin, melanogenesis, and vitiligo. Fortschr Chem Org Naturst. 2007:88:131-85.

22. Dadachova E, Casadevall A. Melanin as a potential target for radionuclide therapy of metastatic melanoma. Future Oncol. 2005;1(4):541-9.

23. Assefa Z, Van Laethem A, Garmyn M, Agostinis P. Ultraviolet radiation-induced apoptosis in keratinocytes: on the role of cytosolic factors. Biochim Biophys Acta. 2005;1755(2):90-106

24. Archambault M, Yaar M, Gilchrest BA. Keratinocytes and fibroblasts in a human skin equivalent model enhance melanocyte survival and melanin synthesis after ultraviolet irradiation. J Invest Dermatol. 1995;104(5):859-67.

25. Boissy RE. Melanosome transfer to and translocation in the keratinocyte. Exp Dermatol. 2003;12 Suppl 2:5-12.

26. Takeuchi S, Zhang W, Wakamatsu K, Ito S, Hearing VJ, Kraemer KH, et al. Melanin acts as a potent UVB photosensitizer to cause an atypical mode of cell death in murine skin. Proc Natl Acad Sci U S A. 2004;101(42):15076-81.

27. Kobayashi N, Muramatsu T, Yamashina Y, Shirai T, Ohnishi T, Mori T. Melanin reduces ultraviolet-induced DNA damage formation and killing rate in cultured human melanoma cells. J Invest Dermatol. 1993;101(5):685-9.

28. Perry PK, Silverberg NB. Cutaneous malignancy in albinism. Cutis. 2001;67(5):427-30

29. Baxter LL, Loftus SK, Pavan WJ. Networks and pathways in pigmentation, health, and disease. Wiley Interdiscip Rev Syst Biol Med. 2009;1(3):359-71.

30. Ho H, Milenkovic T, Memisevic V, Aruri J, Przulj N, Ganesan A. Protein interaction network topology uncovers melanogenesis regulatory network components within functional genomics datasets. BMC Syst Biol. 2010;4(1):84

31. Hearing VJ, Jimenez M. Mammalian tyrosinase-the critical regulatory control point in melanocyte pigmentation. Int J Biochem. 1987;19(12):1141-7.

32. Vetrini F, Auricchio A, Du J, Angeletti B, Fisher DE, Ballabio A, et al. The microphthalmia transcription factor (Mitf) controls expression of the ocular albinism type 1 gene: link between melanin synthesis and melanosome biogenesis. Mol Cell Biol. 2004;24(15):6550-9.

33. Seiberg M, Paine C, Sharlow E, Andrade-Gordon P, Costanzo M, Eisinger M, et al. The protease-activated receptor 2 regulates pigmentation via keratinocyte-melanocyte interactions. Exp Cell Res. 2000;254(1):25-32.

34. Scott GA, Cassidy L. Rac1 mediates dendrite formation in response to melanocyte stimulating hormone and ultraviolet light in a murine melanoma model. J Invest Dermatol. 1998:111(2):243-50.

35. Zhang K, Liu G-H, Yi F, Montserrat N, Hishida T, Esteban C, et al. Direct conversion of human fibroblasts into retinal pigment epithelium-like cells by defined factors. Protein Cell. 2014;5(1):48-58.

36. Di Nuzzo S, Masotti A. Depigmentation therapy in vitiligo universalis with cryotherapy and 4-hydroxyanisole. Clin Exp Dermatol. 2010;35(2):215-6.

37. Dell'Anna ML, Mastrofrancesco A, Sala R, Venturini M, Ottaviani M, Vidolin $A P$, et al. Antioxidants and narrow band-UVB in the treatment of vitiligo: a double-blind placebo controlled trial. Clin Exp Dermatol. 2007;32(6):631-6.

38. Funahashi A, Tanimura N, Morohashi M, Kitano H. Cell designer: a process diagram editor for gene-regulatory and biochemical networks. BIOSILICO. 2003;1:159-62.

39. NetPro [http://www.molecularconnections.com/home/home/products/ netPro].

40. Shannon P, Markiel A, Ozier O, Baliga NS, Wang JT, Ramage D, et al. Cytoscape: a software environment for integrated models of biomolecular interaction networks. Genome Res. 2003;13(11):2498-504.

41. Matlab BGL [http://www.stanford.edu/ dgleich/programs/matlab_bgl].

42. Buckman SY, Gresham A, Hale P, Hruza G, Anast J, Masferrer J, et al. COX-2 expression is induced by UVB exposure in human skin: implications for the development of skin cancer. Carcinogenesis. 1998;19(5):723-9.

43. Kim JY, Shin JY, Kim MR, Hann SK, Oh SH. siRNA-mediated knock-down of COX-2 in melanocytes suppresses melanogenesis. Exp Dermatol. 2012:21(6):420-5.

44. Kamijo M, Nishiyama C, Takagi A, Nakano N, Hara M, Ikeda S, et al. Cyclooxygenase-2 inhibition restores ultraviolet B-induced downregulation of ATP2A2/SERCA2 in keratinocytes: possible therapeutic approach of cyclooxygenase-2 inhibition for treatment of Darier disease. Br J Dermatol. 2012:166(5):1017-22.

45. Smith KA, Tong X, Abu-Yousif AO, Mikulec CC, Gottardi CJ, Fischer SM, et al. UVB radiation-induced beta-catenin signaling is enhanced by COX-2 expression in keratinocytes. Mol Carcinog. 2012;51(9):734-45.

46. Terazawa S, Nakajima H, Shingo M, Niwano T, Imokawa G. Astaxanthin attenuates the UVB-induced secretion of prostaglandin E2 and interleukin-8 in human keratinocytes by interrupting MSK1 phosphorylation in a ROS depletion-independent manner. Exp Dermatol. 2012;21 Suppl 1:11-7.

47. Phung B, Sun J, Schepsky A, Steingrimsson E, Ronnstrand L. C-KIT signaling depends on microphthalmia-associated transcription factor for effects on cell proliferation. PLoS One. 2011;6(8), e24064.

48. Wu LC, Lin YY, Yang SY, Weng YT, Tsai YT. Antimelanogenic effect of c-phycocyanin through modulation of tyrosinase expression by upregulation of ERK and downregulation of p38 MAPK signaling pathways. J Biomed Sci. 2011;18:74.

49. Kim SH, Choi YJ, Moon KM, Lee HJ, Woo Y, Chung KW, et al. The inhibitory effect of a synthetic compound, (Z)-5-(2,4-dihydroxybenzylidene) thiazolidine-2,4-dione (MHY498), on nitric oxide-induced melanogenesis. Bioorg Med Chem Lett. 2013;23(15):4332-5.

50. Kvam E, Tyrrell RM. Induction of oxidative DNA base damage in human skin cells by UV and near visible radiation. Carcinogenesis. 1997;18(12):2379-84.

51. Chakraborty AK, Funasaka Y, Slominski A, Ermak G, Hwang J, Pawelek JM, et al. Production and release of proopiomelanocortin (POMC) derived peptides by human melanocytes and keratinocytes in culture: regulation by ultraviolet B. Biochim Biophys Acta. 1996;1313(2):130-8.

52. Fabrikant J, Touloei K, Brown SM. A review and update on melanocyte stimulating hormone therapy: afamelanotide. J Drugs Dermatol. 2013;12(7):775-9.

53. Wang WQ, Wu JF, Xiao XQ, Xiao Q, Wang J, Zuo FG. Narrow-band UVB radiation promotes dendrite formation by activating Rac1 in B16 melanoma cells. Mol Clin Oncol. 2013;1(5):858-62.

54. Wu CS, Lan CC, Kuo HY, Chai CY, Chen WT, Chen GS. Differential regulation of nuclear factor-kappa B subunits on epidermal keratinocytes by ultraviolet B and tacrolimus. Kaohsiung J Med Sci. 2012:28(11):577-85.

55. Ahmad I, Jimenez H, Yaacob NS, Yusuf N. Tualang honey protects keratinocytes from ultraviolet radiation-induced inflammation and DNA damage. Photochem Photobiol. 2012;88(5):1198-204.

56. Oh CT, Park Jl, Jung YR, Joo YA, Shin $\mathrm{DH}$, Cho HJ, et al. Inhibitory effect of Korean Red Ginseng on melanocyte proliferation and its possible implication in GM-CSF mediated signaling. J Ginseng Res. 2013;37(4):389-400.

57. Tang L, Li J, Lin X, Wu W, Kang K, Fu W. Oxidation levels differentially impact melanocytes: low versus high concentration of hydrogen peroxide promotes melanin synthesis and melanosome transfer. Dermatology. 2012;224(2):145-53

58. Jung E, Lee JA, Shin S, Roh KB, Kim JH, Park D. Madecassoside inhibits melanin synthesis by blocking ultraviolet-induced inflammation. Molecules 2013;18(12):15724-36.

59. Imokawa G, Kobayashi T, Miyagishi M, Higashi K, Yada Y. The role of endothelin-1 in epidermal hyperpigmentation and signaling mechanisms of mitogenesis and melanogenesis. Pigment Cell Res. 1997;10(4):218-28.

60. Hirobe T. Stimulation of dendritogenesis in the epidermal melanocytes of newborn mice by melanocyte-stimulating hormone. J Cell Sci. 1978;33:371-83

61. Seiberg M, Paine C, Sharlow E, Andrade-Gordon P, Costanzo M, Eisinger M, et al. Inhibition of melanosome transfer results in skin lightening. J Invest Dermatol. 2000:115(2):162-7.

62. Marwaha V, Chen YH, Helms E, Arad S, Inoue H, Bord E, et al. T-oligo treatment decreases constitutive and UVB-induced COX-2 levels through p53- and NFkappaB-dependent repression of the COX-2 promoter. J Biol Chem. 2005;280(37):32379-88. 